\title{
Changes in the Macular Vascular Density After Bariatric Surgery Measured by Optical Coherence Tomography Angiography
}

\author{
Malak EIShazly \\ Marwa Salama \\ Kareem Elessawy \\ Ophthalmology Department, Cairo \\ University, Cairo, Egypt
}

Correspondence: Kareem Elessawy Ophthalmology Department, Cairo University, 3 Toman Bay Street, Ibn Sandr Square, Msr Elgdida, Cairo, Egypt

Email kemos1984@yahoo.com
Purpose: To evaluate the effect of the weight loss, 3 months after bariatric surgery, on the macular thickness and macular vascular density by optical coherence tomography angiography (OCTA).

Methods: Forty obese patients were included in this prospective study. Body mass index (BMI), macular thickness (whole, fovea, parafovea and perifovea), macular vascular density (VD) in superficial capillary plexus (whole, fovea, parafovea and perifovea), and macular vascular density in deep capillary plexus (whole, fovea, parafovea and perifovea) were measured before and 3 months after bariatric surgery.

Results: The BMI was significantly reduced postoperatively to $43.75 \pm 4.4 \mathrm{~kg} / \mathrm{m}^{2}$ compared to the preoperative results, $55.31 \pm 5.1 \mathrm{~kg} / \mathrm{m}^{2}(p<0.05)$. There was significant increase in macular thickness in the fovea and parafovea postoperatively $(p<0.05)$ but no significant increase in the perifovea. There was significant increase in macular vascular density in the deep capillary plexus postoperatively $(p<0.05)$ but no significant increase in the macular vascular density in the superficial capillary plexus postoperatively $(p>0.05)$. Significant correlations were detected between the BMI changes and changes in different macular parameters.

Conclusion: Bariatric surgery showed significant effect on certain indices in macular thickness and macular vascular density, especially in the deep capillary plexus. Therefore, OCTA is considered a valuable tool to assess the short-term changes in macular microcirculation following significant weight reduction.

Keywords: bariatric surgery, optical coherence tomography angiography, OCTA, macular thickness, macular vessel density

\section{Introduction}

Obesity is a major health problem resulting in serious cardiovascular diseases, diabetes mellitus and musculoskeletal disorders. ${ }^{1}$ It affects the function of many organ systems, the pathogenesis of which may be mechanical, vascular or oxidative stress. ${ }^{2}$

Obesity is a risk factor in different ocular diseases, including age-related macular degeneration, senile cataract and diabetic retinopathy, with the underlying pathology at the microvasculature level. ${ }^{3}$

The weight loss after bariatric surgery was found to have a beneficial effect on different diseases associated with obesity, including the eye. ${ }^{4}$ Recent studies show significant effect of weight reduction on retinal thickness and retinal microvascular perfusion. ${ }^{5,6}$ 
Optical coherence tomography angiography (OCTA) is a non-invasive technique which enables detailed morphological visualization to quantitatively assess and detect any abnormalities in the retinal microvasculature, including those in the macular region. ${ }^{7}$

The aim of this study was to evaluate the effect of weight loss after bariatric surgery on the macular vessel density (VD) and macular thickness in patients who had undergone bariatric surgery, three months postoperatively, using OCTA.

\section{Patients and Methods}

A total of 40 obese patients with a body mass index (BMI) of $>45 \mathrm{~kg} / \mathrm{m}^{2}$ were recruited and scheduled for bariatric surgery. They were prospectively enrolled from June 2018 to January 2020.

\section{Ethical Statement}

This study adhered to international ethical standards and tenants of the Declaration of Helsinki. Participants were informed about the study objectives, methodology, risk, and benefit. Then, written informed consent was obtained from recruited patients before enrollment in the study. Our protocol was reviewed and then approved by the ethics committee of El Nour eye hospital.

Only patients eligible for bariatric surgery as defined by the World Health Organization were included in this study. Other inclusion criteria were age $>18$ years, $6 / 6$ best corrected visual acuity (BCVA) by Snellen chart, spherical equivalent between +1 and -4 diopters (D), intraocular pressure $(\mathrm{IOP})<21 \mathrm{mmHg}$ and clear refractive media allowing sufficient image quality.

Patients with retinal or systemic vascular disorders, any associated ocular pathology (eg macular degeneration, glaucoma or uveitis), prior ophthalmic surgery or trauma were excluded.

Complete physical examination of the patients, history taking and anthropometric measurements including weight and height, fasting blood sugar and blood pressure measurements were done at the bariatric unit of the hospital. Patients with high blood sugar levels or high blood pressure measurements were excluded.

All subjects underwent a comprehensive ophthalmological examination, including BCVA, refraction and IOP measurement by Goldmann applanation tonometer. Anterior and posterior segment examinations were performed to rule out any ocular pathology.

Optical coherence tomography (OCT) and OCTA images of the macular region were done for all patients with the AngioVue system (Optovue RTVue XR Avanti; Optovue, Inc., Fremont, CA, USA). The imaging procedure was done as described previously by Rao et al. ${ }^{8}$ The system has an A-scan rate of 70,000 scans per second using a light source centered on $840 \mathrm{~nm}$ and a bandwidth of $45 \mathrm{~nm}$. Imaging is performed using a set of 2 scans; one vertical priority scan and one horizontal priority raster volumetric scan. The macular scan was performed using volumetric scans covering $3 \times 3 \mathrm{~mm}$. The split-spectrum amplitude decorrelation angiography (SSADA) algorithm was used to extract the OCTA information. Macular vessel densities were analyzed over a $1.5 \mathrm{~mm}$ wide parafoveal, circular annulus centered on the macula, as shown in Figures 1 and 2.

Vessel density is defined as the percentage area occupied by the large vessels and microvasculature in a certain region. Image quality was assessed for all OCTA and OCT scans and the average of 3 readings obtained was used for the statistical analysis. Poor quality images, such as those with a signal strength index (SSI) of $<45$ or images with segmentation errors and motion artifacts, were excluded from the analysis. One eye from each patient (the left eye) was imaged and analyzed.

The bariatric surgery performed was gastric bypass surgery in which the stomach was divided into a small upper pouch and a larger lower pouch (remnant), and then the small intestine was connected to both.

The BMI, calculated as weight $(\mathrm{kg}) /$ height $^{2}\left(\mathrm{~m}^{2}\right)$, OCT and OCTA imaging analysis were performed before and 3 months post-bariatric surgery.

Statistical analysis was done using IBM SPSS v20.0 statistical software (IBM Corporation, New York, USA). Descriptive statistics were calculated and the data was summarized as mean \pm standard deviation $( \pm \mathrm{SD})$, median and range, or frequencies (number of cases) and percentages when appropriate. Comparison of numerical variables was done using independent samples student's $t$-test. Comparisons between preoperative data and postoperative data were carried out using paired-samples $t$-test. Correlation between the changes in different variables was done using Pearson's correlation coefficient. The results were considered statistically significant with a $p$-value of $\leq 0.05$.

\section{Results}

A total of 40 eyes of 40 obese patients (19 males, 21 females) seeking bariatric surgery were included in this study. Mean age was $35.7 \pm 3.4$ years. Three months postoperatively, the mean BMI was significantly reduced to 

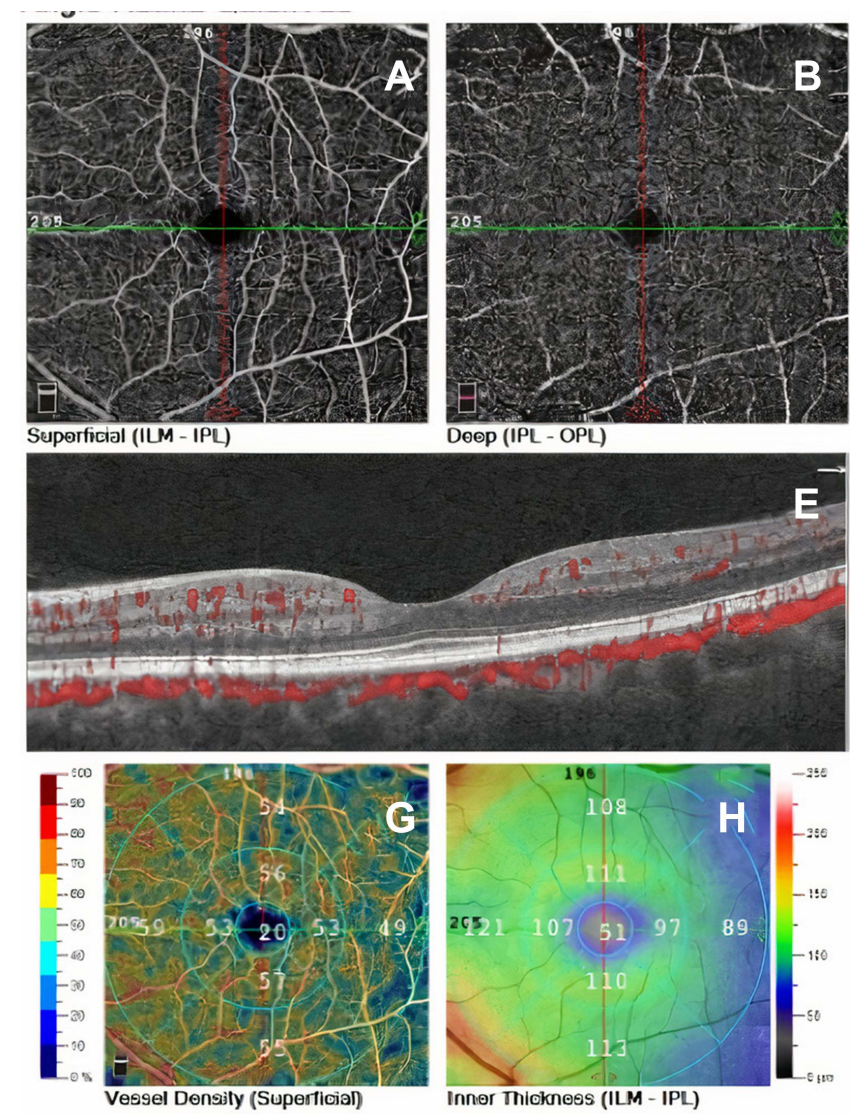

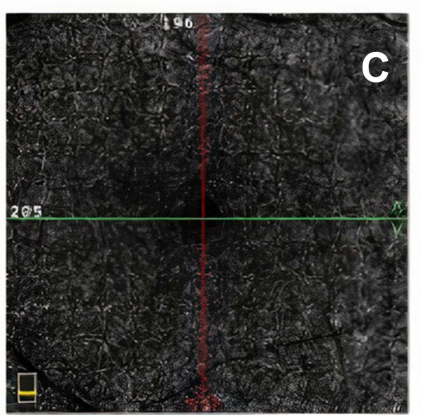

Ouler Relina (OPL - BRM)
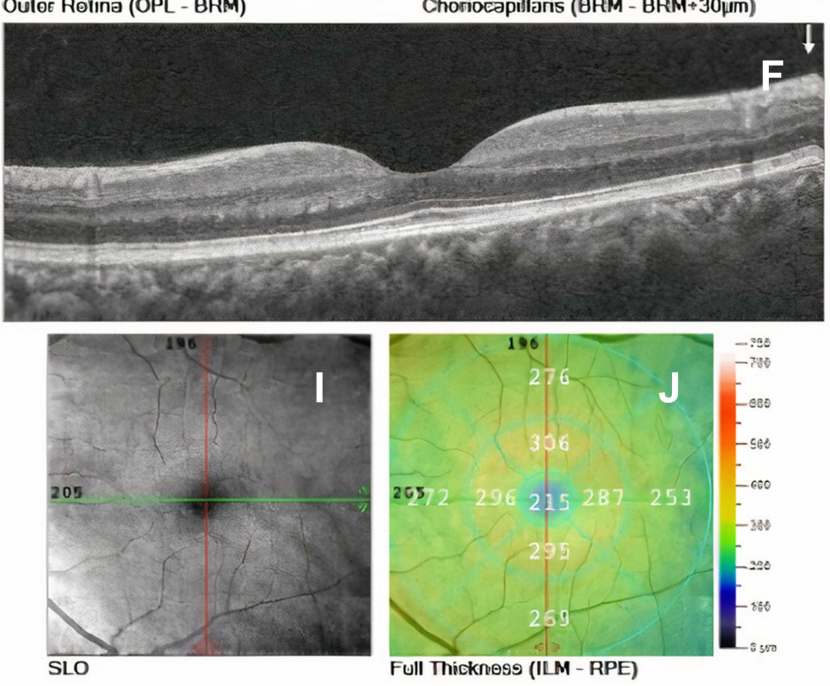

Figure I Optical coherence tomography angiography images of the left eye of an included patient showing superficial and deep plexuses (A and B), outer retina (C), choriocapillaris (D), cross-sectional B-scans (E and F) with angio overlay (E), vessel density (superficial) (G), inner thickness (H), scanning laser ophthalmoscopy (I) and full thickness images $(\mathbf{J})$.

$43.75 \pm 4.4 \mathrm{~kg} / \mathrm{m}^{2}(p<0.05)$ compared to the preoperative value of $51.31 \pm 5.1 \mathrm{~kg} / \mathrm{m}^{2}$. The mean preoperative and 3 months postoperative changes in macular thickness with their significance are detailed in Table 1.

A significant statistical increase in macular thickness areas of the fovea and parafovea (Average, Superior-Hemi and Inferior-Hemi) was detected 3 months after bariatric surgery $(p<0.05)$. However, there was no significant statistical increase in the whole macular thickness area and areas of perifovea (Average, Superior-Hemi and Inferior-Hemi) 3 months after the surgery $(p>0.05)$. The decrease in the foveal avascular zone (FAZ) area was statistically insignificant too $(p>0.05)$.

The mean preoperative and 3 months postoperative changes in macular VD in superficial and deep plexus with their significance are described in Table 2 . Significant statistical increase in macular VD was detected in the deep capillary plexus 3 months after the bariatric surgery $(p<0.05)$. Meanwhile, the macular VD in the superficial capillary plexus showed no significant difference $(p>0.05)$.
Statistically significant correlations between the BMI and macular thickness, VD in different areas and the FAZ area were found and are shown in Table 3.

\section{Discussion}

In this study, 40 obese patients were investigated for the effect of bariatric surgery on the macular thickness and macular vascular density in both superficial and deep plexuses. The macular thickness in whole macula in our study preoperatively was $274.55 \pm 7.9 \mu \mathrm{m}$ and central macular thickness preoperatively at the fovea was 231.35 $\pm 19.2 \mu \mathrm{m}$ with mean BMI of $55.31 \pm 5.1 \mathrm{~kg} / \mathrm{m}^{2}$.

In 2016, Dogan et al studied a group of 67 patients defined as having morbid or class III obesity with mean BMI of $49.15 \pm 7.65 \mathrm{~kg} / \mathrm{m}^{2}$ and CMT of $240.99 \pm 21.47 \mu \mathrm{m}$, and compared them with 29 age- and sex-matched nonobese individuals with mean BMI of $22.99 \pm 1.89 \mathrm{~kg} / \mathrm{m}^{2}$ and CMT of $240.99 \pm 21.47 \mu \mathrm{m}$. No significant statistical difference of CMT was detected $(p>0.05)$. CMT was 

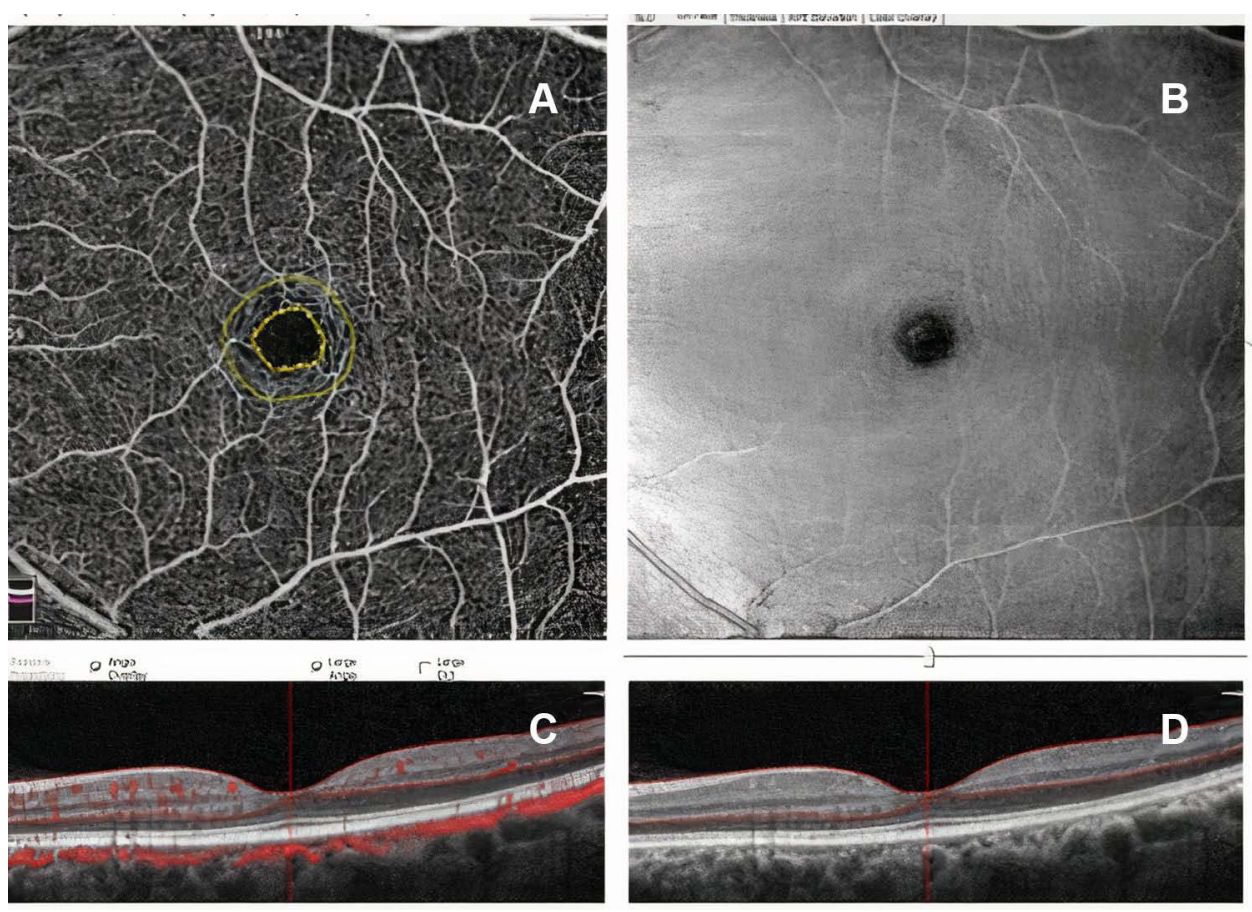

Figure 2 Optical coherence tomography angiography (A), en face (B) and cross-sectional B-scan with segmentation and angio overlay (C and D) images of the left eye of the same patient preoperatively showing the FAZ area.

measured using spectral domain OCT (SD-OCT) (Cirrus HD OCT, Carl Zeiss Meditec, Dublin, CA, USA). ${ }^{9}$

The CMT in our study preoperatively was less than the CMT of the obese group in Dogan's study; this is

Table I The Mean Preoperative and 3 Months Postoperative Changes in Macular Thickness

\begin{tabular}{|l|c|c|c|}
\hline & Preoperative & Postoperative & p-value \\
\hline \multicolumn{4}{|c|}{ Macular thickness $(\mu \mathrm{m}):$} \\
Wovea & $\begin{array}{c}274.55 \pm 7.9 \\
231.35 \pm 19.2\end{array}$ & $\begin{array}{c}274.60 \pm 9.1 \\
232.40 \pm 19.4\end{array}$ & $\begin{array}{c}0.9 \\
0.002^{*}\end{array}$ \\
\hline \multicolumn{4}{|c|}{ Parafovea $(\mu \mathrm{m}):$} \\
\hline Average & $311.35 \pm 14.9$ & $313.01 \pm 16.2$ & $0.000 I^{*}$ \\
Superior-Hemi & $311.45 \pm 14.5$ & $312.90 \pm 15.7$ & $0.00 I^{*}$ \\
Inferior-Hemi & $310.95 \pm 15.9$ & $313.01 \pm 17.0$ & $0.000 I^{*}$ \\
\hline \multicolumn{5}{|c|}{ Perifovea $(\mu \mathrm{m}):$} \\
\hline Average & $271.95 \pm 7.8$ & $272.01 \pm 8.8$ & 0.9 \\
Superior-Hemi & $273.10 \pm 8.4$ & $273.10 \pm 9.3$ & 0.9 \\
Inferior-Hemi & $270.90 \pm 8.2$ & $271.05 \pm 9.2$ & 0.8 \\
\hline \multicolumn{5}{|c|}{ FAZ area $\left(\mathrm{mm}^{2}\right):$} \\
\hline \multicolumn{2}{|c|}{$0.32 \pm 0.1$} & $0.31 \pm 0.1$ & 0.3 \\
\hline
\end{tabular}

Note: *With statistical significance.

Abbreviations: $\mu \mathrm{m}$, micrometer; $\mathrm{mm}^{2}$, millimeter square. explained by the fact that our mean BMI was higher than theirs. ${ }^{9}$

In the current study, a significant increase in the macular thickness areas of fovea and parafovea (Average, Superior-Hemi and Inferior-Hemi) was detected 3 months after bariatric surgery $(p<0.05)$. However, there was no significant increase in the macular thickness area and areas of perifovea (Average, Superior-Hemi and Inferior-Hemi) 3 months after the surgery ( $p>0.05)$.

Our results were similar to Brynskov et al, in which the retinal thickness of 51 patients with type 2 diabetes with no diabetic retinopathy was examined 2 weeks before and 1, 3, 6 and 12 months after bariatric surgery using SD OCT (Heidelberg Spectralis, Heidelberg Engineering $\mathrm{GmbH}$, Heidelberg, Germany). Their study showed that a significant increase in macular thickness (total macula, fovea, parafovea and perifovea) started at the 3rd postoperative month, peaked at the 6th month, then dropped back after 12 months. ${ }^{10}$

Also, Laiginhas et al reported significant increase of the retinal thickness after bariatric surgery but without change in peripapillary RNFL thickness and it was attributed to improved perfusion after weight reduction. ${ }^{5}$

OCTA is an emerging non-invasive tool for imaging and quantifying retinal vasculature at the level of both superficial and deep plexuses and areas of non-perfusion 
Table 2 The Mean Preoperative and 3 Months Postoperative Changes in Macular Vascular Density

\begin{tabular}{|c|c|c|c|}
\hline $\begin{array}{l}\text { Macular Vessel } \\
\text { Density }\end{array}$ & Preoperative & Postoperative & $p$-value \\
\hline \multicolumn{4}{|c|}{ Deep VD \%: } \\
\hline Whole & $53.28 \pm 5.2$ & $55.12 \pm 3.8$ & $0.008^{*}$ \\
\hline Fovea & $33.6 I \pm 6.5$ & $36.07 \pm 6.3$ & $0.001 *$ \\
\hline \multicolumn{4}{|c|}{ Parafovea } \\
\hline Average & $56.19 \pm 4.1$ & $59.38 \pm 3.2$ & $0.01 *$ \\
\hline Superior-Hemi & $58.69 \pm 3.9$ & $59.87 \pm 3.3$ & $0.008^{*}$ \\
\hline Inferior-Hemi & $57.73 \pm 4.8$ & $59.90 \pm 3.3$ & $0.04^{*}$ \\
\hline \multicolumn{4}{|c|}{ Perifovea: } \\
\hline Average & $55.16 \pm 5.7$ & $57.17 \pm 4.1$ & $0.007^{*}$ \\
\hline Superior-Hemi & $54.87 \pm 5.5$ & $56.7 I \pm 4.1$ & $0.01 *$ \\
\hline Inferior-Hemi & $55.47 \pm 6.2$ & $57.64 \pm 4.4$ & $0.01 *$ \\
\hline \multicolumn{4}{|c|}{ Superficial VD \%: } \\
\hline Whole & $51.79 \pm 2.6$ & $52.05 \pm 1.9$ & 0.4 \\
\hline Fovea & $|8.4| \pm 5.5$ & $19.06 \pm 5.6$ & 0.3 \\
\hline \multicolumn{4}{|c|}{ Parafovea } \\
\hline Average & $55.09 \pm 3.6$ & $55.72 \pm 1.9$ & 0.3 \\
\hline Superior-Hemi & $55.04 \pm 3.7$ & $55.79 \pm 1.9$ & 0.1 \\
\hline Inferior-Hemi & $55.03 \pm 4.1$ & $55.63 \pm 1.9$ & 0.5 \\
\hline \multicolumn{4}{|c|}{ Perifovea } \\
\hline Average & $52.32 \pm 2.6$ & $52.58 \pm 2.3$ & 0.4 \\
\hline Superior-Hemi & $52.23 \pm 2.4$ & $52.25 \pm 2.5$ & 0.6 \\
\hline Inferior-Hemi & $52.38 \pm 3.1$ & $53.02 \pm 2.3$ & 0.3 \\
\hline
\end{tabular}

Note: *With statistical significance.

Abbreviation: VD, vessel density.

such as FAZ. Utilizing standard sectors to measure the VD in the macular and the peripapillary regions allows the application of a uniform method to measure the VD in different ophthalmological conditions. ${ }^{11}$

In normal subjects, it was found that the macular VD in the deep plexus was higher than in the superficial retinal plexuses. $^{11}$

The current study also showed that preoperative macular VD in the deep plexus is higher than in the superficial plexus in all macular regions. This finding was not far from that of Zhang et al, who studied the VD before intraocular pressure changes. ${ }^{12}$

To our knowledge this research was the first to study the effect of weight loss after bariatric surgery on the blood flow of the macular region by measuring the VD in both deep and superficial capillary plexuses in different macular areas by using OCTA AngioVue system (Optovue RTVue XR Avanti; Optovue, Inc., Fremont, CA, USA).

We detected significant statistical increase in macular VD in the deep capillary plexus of all macular areas 3 months after bariatric surgery $(p<0.05)$. However, the macular VD in the superficial capillary plexus in all macular areas did not show significant difference $(p>0.05)$.

Our results were similar to Laiginhas et al, who reported improvement in retinal perfusion after gastric bypass surgery, as demonstrated by the increased VD in the deep capillary plexus in the parafoveal region. ${ }^{6}$

Significant correlations were detected in this study between changes in BMI 3 months postoperatively and changes in the macular thickness in certain areas, as shown in Table 3.

The significant improvement in the macular VD of the deep plexus in our results can be explained by Çekiç et al, who showed the effect of bariatric surgery using sleeve gastrectomy on the retrobulbar flow hemodynamic parameters. They found a significant increase in the central retinal artery and the ophthalmic artery blood flow measured by color Doppler ultrasound 6 months, after a significant BMI reduction of $12.5 \mathrm{~kg} / \mathrm{m}^{2} .^{13}$

The insignificant improvement of the VD in the superficial plexus in all macular areas in the current study may be attributed to Shoji et al, who stated that vascular measurements obtained by OCTA only reflect some aspects of blood flow within the detected vessels, and do not represent an estimate of real blood flow. Specifically, this modality detects vasculature based on amplitude decorrelation, which results from perfused vessels, but does not directly quantify the flow rate within the detected vessels. ${ }^{14}$

The discrepancy in the changes in the superficial and deep plexus was observed in many studies; it was explained that the deep capillary plexus is more sensitive to pathological conditions than the superficial ones. ${ }^{15-17}$

This study is not free from limitations. In addition to the general limitations related to the OCTA technology itself, ${ }^{18}$ the short postoperative follow-up period that may prevent detection of other changes or more significant improvements in superficial plexus VD indices is an additional downside; hence further studies with longer follow-up are needed. Also, it represented the 
Table 3 Correlations Between Changes in BMI and Changes in Optical Coherence Tomography and Optical Coherence Tomography Angiograph

\begin{tabular}{|c|c|c|c|}
\hline \multicolumn{4}{|c|}{ Correlations Between: } \\
\hline & Changes in & $\boldsymbol{r}$ & $p$-value \\
\hline \multirow{25}{*}{$\begin{array}{l}\text { Changes in } \\
\text { BMI }\end{array}$} & Deep Whole VD & 0.289 & $0.025^{*}$ \\
\hline & Deep Fovea VD & 0.203 & 0.12 \\
\hline & Deep Parafovea Average VD & 0.166 & 0.205 \\
\hline & Deep Parafovea Superior-Hemi VD & 0.18 & 0.168 \\
\hline & Deep Parafovea Inferior-Hemi VD & 0.281 & $0.03^{*}$ \\
\hline & Deep Perifovea Average VD & 0.254 & $0.05^{*}$ \\
\hline & Deep Perifovea Superior-Hemi VD & 0.125 & $0.34 I$ \\
\hline & Deep Perifovea Inferior-Hemi VD & 0.242 & 0.06 \\
\hline & Superficial Whole VD & -0.02 & 0.881 \\
\hline & Superficial Fovea VD & -0.21 & 0.108 \\
\hline & Superficial Parafovea Average VD & 0.024 & 0.854 \\
\hline & Superficial Parafovea Superior-Hemi VD & -0.085 & 0.519 \\
\hline & Superficial Parafovea Inferior-Hemi VD & 0.127 & 0.333 \\
\hline & Superficial Perifovea Average VD & -0.13 & 0.321 \\
\hline & Superficial Perifovea Superior-Hemi VD & -0.21 & 0.107 \\
\hline & Superficial Perifovea Inferior-Hemi VD & -0.23 & 0.863 \\
\hline & Thickness Whole & -0.251 & 0.053 \\
\hline & Thickness Fovea & -0.093 & 0.48 \\
\hline & Thickness Parafovea Average & -0.206 & 0.114 \\
\hline & Thickness Parafovea Superior-Hemi & -0.159 & 0.226 \\
\hline & Thickness Parafovea Inferior-Hemi & -0.383 & $0.003 *$ \\
\hline & Thickness Perifovea Average & -0.297 & $0.021^{*}$ \\
\hline & Thickness Perifovea Superior-Hemi & -0.354 & $0.006 *$ \\
\hline & Thickness Perifovea Inferior-Hemi & -0.215 & 0.099 \\
\hline & FAZ area & 0.333 & $0.009 *$ \\
\hline
\end{tabular}

Note: *With statistical significance.

Abbreviations: BMI, body mass index; VD, vessel density.

results of a single center only, with a relatively homogenous population with certain ethnic origin and it did not put the gender of the patients into consideration.

In conclusion, bariatric surgery had a significant effect on some indices of macular VD measured by OCTA in obese patients 3 months postoperatively. OCTA can therefore be considered a valuable tool to assess the shortterm changes in the retinal microcirculation following significant weight reduction.

\section{Data Sharing Statement}

All data are available upon request. 


\section{Ethical Statement}

This study adhered to international ethical standards and tenants of the Declaration of Helsinki. Participants were informed about the study objectives, methodology, risk, and benefit. Then, written informed consent was obtained from recruited patients before enrollment in the study. Our protocol was reviewed and then approved by the ethics committee of El Nour eye hospital.

\section{Consent to Participate}

Informed consent was obtained from every patient before participation in the study.

\section{Consent to Publish}

Informed consent was obtained to publish their data.

\section{Author Contributions}

All authors made a significant contribution to the work reported, whether that is in the conception, study design, execution, acquisition of data, analysis and interpretation, or in all these areas; took part in drafting, revising or critically reviewing the article; gave final approval of the version to be published; have agreed on the journal to which the article has been submitted; and agree to be accountable for all aspects of the work.

\section{Disclosure}

The authors report no conflicts of interest in this work.

\section{References}

1. Haslam DW, James WP. Obesity. Lancet. 2005;366(9492):1197-1209.

2. Cheung N, Wong TY. Obesity and eye diseases. Surv Ophthalmol. 2007;52:180-195.

3. Zhang QY, Tie LJ, Wu SS. Overweight, obesity, and risk of age related macular degeneration. Invest Ophthalmol Vis Sci. 2016;57:1276-1283.
4. Eliash ZB, Achiron A, Hecht I, Shimonov M. Reduction of intraocular pressure after bariatric surgery. Acta Ophthalmol. 2018;96(5): e592-e595.

5. Laiginhas R, Guimarães M, Cardoso P, et al. Bariatric surgery induces retinal thickening without affecting the retinal nerve fiber layer independent of diabetic status. Obes Surg. 2020;30(12):4877-4884.

6. Laiginhas R, Guimarães M, Nora M, Chibante J, Falcão M. Gastric Bypass improves microvascular perfusion in patients with obesity. Obes Surg. 2021;8:1-7.

7. Nagiel A, Sadda SR, Sarraf D. A promising future for optical coherence tomography angiography. JAMA Ophthalmol. 2015;133:629-630.

8. Rao HL, Pradhan ZS, Weinreb RN. Regional comparisons of optical coherence tomography angiography vessel density in primary open-angle glaucoma. Am J Ophthalmol. 2016;171:75-83.

9. Dogan B, Kazim Erol M, Dogan U, et al. The retinal nerve fiber layer, choroidal thickness, and central macular thickness in morbid obesity: an evaluation using spectral-domain optical coherence tomography. Eur Rev Med Pharmacol Sci. 2016;20(5):886-891.

10. Brynskov T, Laugesen CS, Floyd AK, Sørensen TL. Thickening of inner retinal layers in the parafovea after bariatric surgery in patients with type 2 diabetes. Acta Ophthalmol. 2016;94:668-674.

11. Hassa M, Sadiq MA, Halim MS, et al. Evaluation of macular and peripapillary vessel flow density in eyes with no known pathology using optical coherence tomography angiography. Int J Retin Vitr. 2017;3:27.

12. Zhang Q, Jonas JB, Wang Q, et al. Optical coherence tomography angiography vessel density changes after acute intraocular pressure elevation. Sci Rep. 2018;8:6024.

13. Çekiç B, Dogan B, Toslak I, Dogan U, Saglik S, Erol M. The effect of bariatric surgery on the retrobulbar flow hemodynamic parameters in patients with obesity: color Doppler evaluation. Int Ophthalmol. 2018;38(5):1845-1850.

14. Shoji T, Zangwill LM, Akagi T, et al. Progressive macula vessel density loss in primary open angle glaucoma: a longitudinal study. Am J Ophthalmol. 2017;182:107-117.

15. Lavia C, Bonnin S, Maule M, Erginay A, Tadayoni R, Gaudric A. Vessel density of superficial, intermediate, and deep capillary plexus using optical coherence tomography angiography. Retina. 2019;39 (2):247-258.

16. Iafe NA, Phasukkijwatana N, Chen X, Sarraf D. Retinal capillary density and foveal avascular zone area are age-dependent: quantitative analysis using optical coherence tomography angiography. Invest Ophthalmol Vis Sci. 2016;57:5780-5787.

17. Coscas F, Sellam A, Glacet-Bernard A, et al. Normative data for vascular density in superficial and deep capillary plexuses of healthy adults assessed by optical coherence tomography angiography. Invest Ophthalmol Vis Sci. 2016;57:OCT211-223.

18. Sambhav K, Grover S, Chalam K. The application of optical coherence tomography angiography in retinal diseases. Surv Ophthalmol. 2017;62(6):838-866.
Clinical Ophthalmology

\section{Publish your work in this journal}

Clinical Ophthalmology is an international, peer-reviewed journal covering all subspecialties within ophthalmology. Key topics include: Optometry; Visual science; Pharmacology and drug therapy in eye diseases; Basic Sciences; Primary and Secondary eye care; Patient Safety and Quality of Care Improvements. This journal is indexed on PubMed
Central and CAS, and is the official journal of The Society of Clinical Ophthalmology (SCO). The manuscript management system is completely online and includes a very quick and fair peer-review system, which is all easy to use. Visit http://www.dovepress.com/ testimonials.php to read real quotes from published authors. 